\title{
AIR POLLUTION ESTIMATION USING AEROSOL OPTICAL THICKNESS BY OLI IMAGES IN TEHRAN
}

\author{
S. M. Mozafari ${ }^{1}$, M. Hasanlou ${ }^{1, *}$, H. Arefi ${ }^{1}$ \\ ${ }^{1}$ School of Surveying and Geospatial Engineering, College of Engineering, University of Tehran \\ (s.mohamadmozafari, hasanlou, hossein.arefi) @ut.ac.ir
}

Commission VI, WG VI/4

KEY WORDS: Air pollution, particulate materials, Aerosol Optical Depth, Landsat-8, OLI, TOA reflectance

\begin{abstract}
:
Air pollution is one of the main problems in human and environmental health in big cities and in developing countries. The precise monitoring and predicting of air quality and the assessing of the amount of contaminants will reduce the risks to human and the environment health. The particulate materials in the atmosphere are divided into two PM2.5 (particulate materials with a diameter of less than $2.5 \mu \mathrm{m}$ ) and PM10 (particulate materials with a diameter of less than $10 \mu \mathrm{m}$ ) groups, the main contributor to the air pollution associated with these pollutants. In this study, an experimental relationship is established between in-situ values of PM2.5 and PM10 with satellite images and a high-precision air pollution model is produced. Also, the impact of some urban parameters such as vegetation on air pollution have been the objectives of this research. Using four year course (From the beginning of 2015 until the end of 2018 from Tehran) from Landsat-8, OLI images and receiving ground data, at the same time, air pollution rates in different parts of Tehran have been investigated. For this purpose, 23 air quality control stations in Tehran have been used. The study suggests that the study of atmospheric reflectance from Landsat-8, OLI is a good alternative to monitoring the quality of air on Earth. The feasibility of the proposed algorithms was investigated based on the correlation coefficient $(\mathrm{R})$ and root mean-square error (RMSE) and normalize root mean-square error (NRMSE) compared with the PM2.5 and PM10 in-situ measurement data. A choice of our proposed multispectral model was founded on the highest value R and lowest value of the RMSE and so and lowest value of NRMSE with PM10 in-situ data. The outcomes of this research showed that visible bands of Landsat 8 OLI were capable of calculating PM2.5 and PM10 concentration to an acceptable level of accuracy.
\end{abstract}

\section{INTRODUCTION}

Air pollution is of concern to many countries around the world. Iran is a developing country, the urbanization process is fast in many cities of Iran, construction in recent years, advances in industrial areas, traffic in these cities is such that the pollution of the air rises. In fact, greenhouse gas emissions from vehicles, thermal power plants and industrial plants are the main sources of atmospheric pollutants.

In general, air pollution in the Earth's atmosphere is caused by contaminants such as $\mathrm{CO}, \mathrm{O}_{3}, \mathrm{NO}_{\mathrm{x}}$ and particulate matter. Suspended particles are liquid and solid particles (mostly solids) that float in the air around the earth and are divided into two main classes of PM2.5 \& PM10 that have the most impact on lung cancer among pollutants (Leili, 2008).

According to the World Health Organization (WHO), around five million people worldwide become infected due to air pollution. Of these, about 800,000 people die from respiratory failure. An important point is that about $63 \%$ of deaths occur in Asia (Saleh, and Hasan, 2014).

Meanwhile, there are 4,300 deaths annually in Tehran due to heart and respiratory illnesses. Respiratory diseases in this city have increased by $27 \%$ over the last ten years (tasnim, 2018) However, in the field of air pollution monitoring, it is currently used in Iran to measure air pollution from air quality monitoring stations. It seems that these stations are very low due to the size of the city of Tehran. On the other hand, the cost of maintaining and developing these stations is very high. There is also a high probability of not capturing air quality data at these stations. Also, ground measurements are impractical if measurements are made in large areas or for continuous monitoring. Therefore, they cannot accurately represent the exact location of air pollutants in major cities such as Tehran.

Past studies around the world have shown that the use of satellite imagery can estimate air pollution in the studied environment with high precision.

In general, remote sensing technology provides environmental monitoring and modelling. However, in the field of air pollution, more comprehensive models for generalization can be found all over the world. In remote sensing methods the basis of modeling atmospheric pollutants is the use of an interpolation between ground air station data quality control and satellite data.

In the past, the use of medium spatial resolution sensors such as MODIS was very common. In this paper, however, it has been attempted to increase the accuracy of the proposed model for air pollution by using high spatial resolution images.

On the other hand, access to air quality indicators on a regular basis and for all urban areas can improve urban management policies to control air pollution and create limits for reducing air pollution, and can even be used for the fair development of the city (from the point of view of the centres Important for hospitals and offices).

\footnotetext{
* Corresponding author
} 
Therefore, in this study, the PM2.5 (particulate materials with a diameter of less than $2.5 \mu \mathrm{m}$ ) and PM10 (particulate materials with a diameter of less than $10 \mu \mathrm{m}$ ) in the atmosphere is presented using Landsat-8, OLI Satellite images in Tehran (Brauer, et al. 2012).

\section{SATELLITE AND IN-SITU DATA SETS}

Data were collected from two categories of data. Data from remote from Landsat-8, OLI images and land data, including the exact shape file of the Tehran's streets Ground data of concentration PM2.5 \& PM10.

\subsection{Remote sensing data}

In this study, Landsat-8, OLI images were used for a four-year period course (From the beginning of 2015 until the end of 2018) from Tehran. Landsat-8 Operational Land Imager (OLI) and Thermal Infrared Sensor (TIRS) images consist of nine spectral bands with a spatial resolution of 30 meters for Bands 1 to 7 and 9. The resolution for Band8 (panchromatic) is 15 meters. Thermal bands 10 and 11 are collected at 100 meters, but are resampled to 30 meter in delivering data product (Table 1).

\begin{tabular}{ccc}
\multicolumn{3}{c}{ Table 1. Landsat-8 Spectral Bands } \\
\hline Band name & $\boldsymbol{\mu} \mathbf{m}$ & Resolution(m) \\
\hline 1 & $0.433-0.453$ & 30 \\
2 & $0.450-0.515$ & 30 \\
3 & $0.525-0.600$ & 30 \\
4 & $0.630-0.680$ & 30 \\
5 & $0.845-0.885$ & 30 \\
6 & $1.560-1.660$ & 30 \\
7 & $2.100-2.300$ & 30 \\
8 & $0.500-0.680$ & 15 \\
9 & $1.360-1.390$ & 30 \\
10 & $10.60-11.20$ & 100 \\
11 & $11.50-12.50$ & 100 \\
\hline
\end{tabular}

Tehran is the capital of Iran located at $35.6892^{\circ} \mathrm{N}, 51.3890^{\circ}$ $\mathrm{E}$ (Figure1). Landsat- 8 for this location is at path/row $164 / 35$

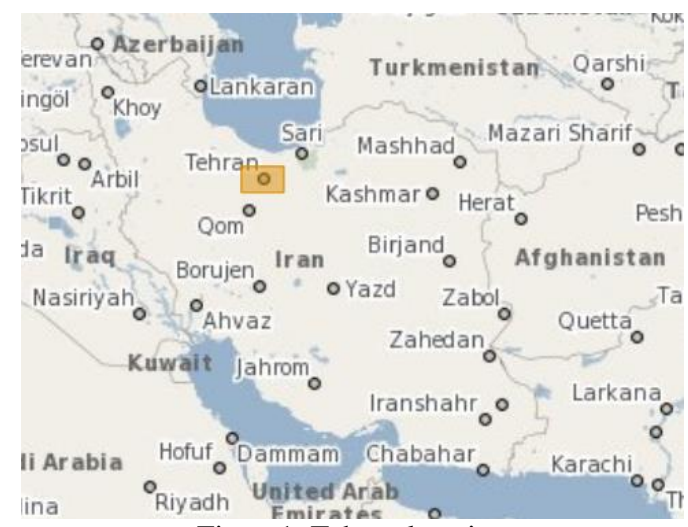

Figure1. Tehran location

\section{2. land data}

Land data, including the exact shape file of the Tehran's streets and data stored in 23 in the city of Tehran by the municipality for a four years' period. Figure 2 shows the study area using the
Ledest- 8 image where the distribution of the municipal air quality control stations is marked in those small yellow circles.

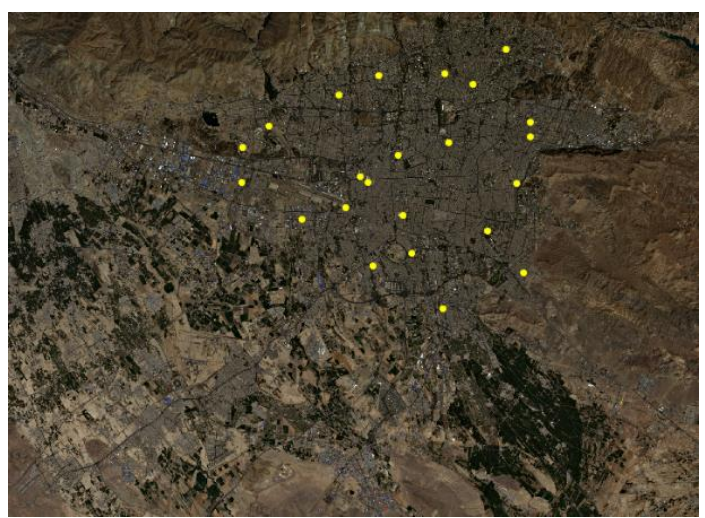

Figure2. study area and station distribution

\section{METHODOLOGY}

The methodology process generally was divided into the following steps: data acquisition, pre-processing, data processing and validation of results. All data preprocessing and processing steps were carried out using MATLAB and ERDAS image and ENVI soft wares.

\section{1. pre-processing}

Data preprocessing includes Radiometric correction, atmospheric correction and geometric correction of images. Also, the calculation of the top of atmosphere reflectance (TOA) is in the next step. In this study, 54 Landsat images were used. After radiometric correction, the following steps were applied to all images.

\subsubsection{Atmospheric Correction}

In order to achieve a relationship between atmospheric correction proposed in this study, the atmospheric FLAASH correction in ENVI software or ATCOR atmospheric correction is in ERDAS image software. [ $\mathrm{n}$ this study, first on the 5 images, FLAASH and ATCOR atmospheric correction were applied simultaneously and given better results for FLAASH atmospheric correction for all other images, FLAASH atmospheric correction was applied.

\subsubsection{Geometric Correction}

After the atmospheric correction is applied to the images. These images are registered with the exact shape file of the Tehran's streets and geometric correction is applied to them.

\subsubsection{TOA Reflectance}

To produce the Aerosol Optical Depth (AOD) Product, the top of atmosphere reflectance (TOA) must be calculated. The top of atmosphere reflectance (TOA) for each image taken separately is obtained using the equation below. The following equation is used to convert DN values to TOA reflectance for OLI data as Eq. 1

$$
p_{\lambda^{\prime}}=M \rho \times Q_{c a l}+A_{\rho}
$$

and TOA reflectance with a correction for the sun angle as Eq.2: 


$$
p_{\lambda}=p_{\lambda^{\prime}}\left(\cos \left(\theta_{S Z}\right)\right)=p_{\lambda^{\prime}} /\left(\sin \left(\theta_{S E}\right)\right)
$$

where, $p_{\lambda^{\prime}}=$ TOA planetary reflectance, without correction for solar angle, $M_{\rho}$ is band specific multiplicative rescaling factor, $A_{\rho}$ is band specific additive rescaling factor, $\mathrm{Q}_{\text {cal }}$ is quantized and calibrated standard product pixel values $(\mathrm{DN}), p_{\lambda}$ is TOA planetary reflectance, with correction for solar angle, $\theta s z$ is local solar zenith angle, and $\theta_{S E}$ is local sun elevation angle.

\subsection{Data processing}

After undergo radiometric correction, the reflectance measured from the satellite (reflectance at the top of atmospheric, TOA) was subtracted by the amount given by the surface reflectance to obtain the atmospheric reflectance.

The Eq. 3 shows the relationship between the atmospheric reflectance with AOD (Hadjimitsis, 2009):

$$
\mathrm{AOD}=\mathrm{a} \times \mathrm{R}(\beta)
$$

where, $R(\beta)$ is atmospheric reflection of the wavelength $(\beta)$ for the satellite. To find the constant coefficients of this equation, we can use the discrete values of PM2.5 and PM10 on the left side of the equation instead of the soil, in which case the following equations are obtained.

$$
\begin{aligned}
& \mathrm{PM} 2.5=\mathrm{a} \times \mathrm{R}(\beta) \\
& \mathrm{PM} 10=\mathrm{b} \times \mathrm{R}(\beta)
\end{aligned}
$$

To get better accuracy, we can use more sentences in the equations.

$$
\begin{aligned}
& \text { PM2.5 }=a 0 \times R(\beta 1)+a 1 \times R(\beta 2)+\ldots \\
& \text { PM10 }=b 0 \times R(\beta 1)+b 1 \times R(\beta 2)+\ldots
\end{aligned}
$$

The atmospheric reflectance was then related to the PM10 using the regression algorithm analysis, from there build calculate models PM2.5 and PM10.

Finally, after producing a high-precision air pollution model, the effect of vegetation parameter on the amount of air pollution is investigated. In this study for achieve to the extent of vegetation the NDVI index was used. The results are presented in three groups: no cover, poor vegetation and good vegetation (Beck, 2011). The Eq. 8 shows the NDVI index for remote sensing images:

$$
\mathrm{NDVI}=(\mathrm{NIR}-\mathrm{VIS}) /(\mathrm{NIR}+\mathrm{VIS})
$$

Where:

VIS $=$ Visible Red

NIR $=$ Near Infrared

And specifically for landsat- 8 the Eq. 9 expressed:

$$
\mathrm{NDVI}=(\mathrm{B} 5-\mathrm{B} 4) /(\mathrm{B} 5+\mathrm{B} 4)
$$

\section{RESULT AND DISCUSSION}

The study found that the greatest impact of atmospheric particulate matter was on Landsat's first to fourth bands. The table. 2 shows the impact of PM10 on the Landsat- 8 bands, and presents a linear binomial relationship between ground observations and remote sensing data for each band. According to this table, the highest correlation is for PM10 with the first band (Coastal aeroso) and the lowest correlation for the fourth band (red).

Table 2. The relationship between atmospheric reflectance of Landsat-8's first to fourth bands with PM10

\begin{tabular}{ccc}
\hline Algorithm & R & RMSE \\
\hline PM10 $=898(\mathrm{R} 1)+28$ & 0.71 & 22.5 \\
PM10=906(R2)+34 & 0.69 & 22.6 \\
PM10=783(R3)+68 & 0.56 & 24.9 \\
PM10=906(R4)+34 & 0.51 & 25.6 \\
& & \\
\hline
\end{tabular}

The table 3 is similar to Table 2 seen for the PM2.5 parameter below, the results of which show that the correlation between PM2.5 and atmospheric reflectance is greater than the correlation between PM10 and atmospheric reflectance.

Table 3. The relationship between atmospheric reflectance of Landsat-8's first to fourth bands with PM2.5

\begin{tabular}{ccc}
\hline Algorithm & R & RMSE \\
\hline PM2.5 $=558(\mathrm{R} 1)+4$ & 0.75 & 8.41 \\
PM10=668 (R2)+5 & 0.89 & 8.33 \\
PM10=685 (R3)+12 & 0.68 & 9.33 \\
PM10= 704(R4)+10.5 & 0.61 & 10.6
\end{tabular}

In the final step of modeling using a linear regression between all bands the following relationships are obtained with the highest accuracy for PM10 \& PM2.5.

PM10 maps were generated using proposed algorithm based on the highest $\mathrm{R}$ and lowest RMSE values, where the highest value of correlation coefficient, $\mathrm{R}$ is 0.82 and the linear regression model as follow is given by Eq. 10 .

$$
\mathrm{PM} 10=-444 \times \mathrm{R}(\beta 1)+1766 \times \mathrm{R}(\beta 3)-1664 \times \mathrm{R}(\beta 4)+78
$$

Also, PM2.5 maps were generated using proposed algorithm based on the highest R and lowest RMSE values, where the highest value of correlation coefficient, $\mathrm{R}$ is 0.879 and the linear regression model as follow is given by Eq. 11:

$$
\text { PM2.5 }=369 \times R(\beta 1)-197 \times R(\beta 3)-376 \times R(\beta d 4)+3.2
$$

The accuracy parameters for the PM10 equations and PM2.5 equations are given in the Table 3.

Table4. accuracy parameters

\begin{tabular}{ccccc}
\hline \multicolumn{2}{c}{ Particulate materials } & $\mathrm{R}$ & $\mathrm{RMSE}$ & $\mathrm{NRMSE}$ \\
\hline \multirow{2}{*}{ PM10 } & Train & 0.90 & 11.9 & 0.119 \\
& $---\mathrm{Test}$ & 0.89 & 22.23 & 0.137 \\
\cline { 2 - 5 } PM2.5 & Train & 0.937 & 5.6 & 0.093 \\
& - Test & 0.87 & 6.6 & 0.14 \\
\hline
\end{tabular}




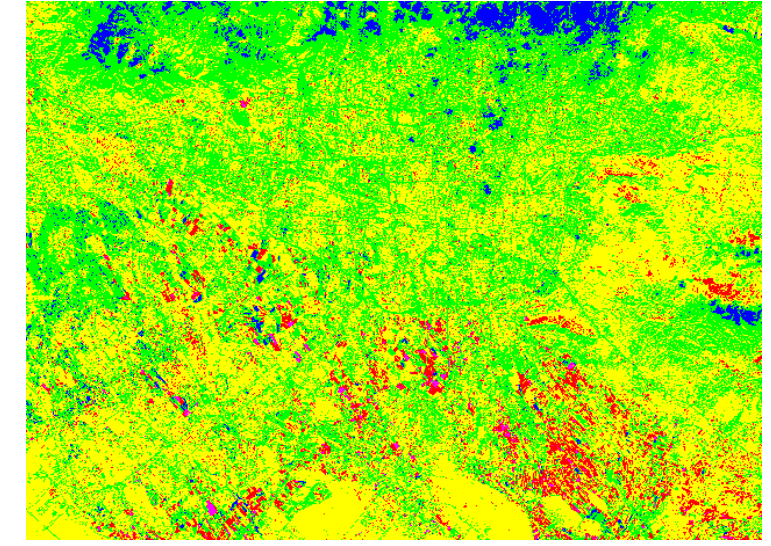

\begin{tabular}{|c|c|}
\hline No data & \\
\hline Clean Air (0-50) & \\
\hline healthy air (50-100) & \\
\hline Unhealthy for sensitive groups (100-150) & \\
\hline Unhealthy (150-200) & \\
\hline Dangerous (>200) &
\end{tabular}

Figure3. PM10 map for January-2018

After producing the contamination model, the effect of vegetation cover was investigated. At this step the two images of lansat- 8 are almost identical in terms of traffic and weather conditions, NDVI vegetation index is applied to both images (figure 4). Figure A is the air pollution map and figure B is the vegetation map of the first image taken in February, and figure $C$ is the air pollution map and figure $\mathrm{D}$ is the vegetation map of the second image. Which was photographed in late March. The sharp decline in vegetation in Tehran's green areas has increased pollution in areas with high potential for air pollution. This comparison shows that if vegetation increased by up to seventy percent, the amount of pollutants in the atmosphere (especially particle material) decreases from average $120 \mu \mathrm{g} / \mathrm{m} 3$ (risk zone for sensitive groups) to $65 \mu \mathrm{g} / \mathrm{m} 3$ (safe zone).

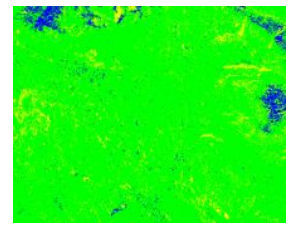

Figure (a) is PM10 map on February

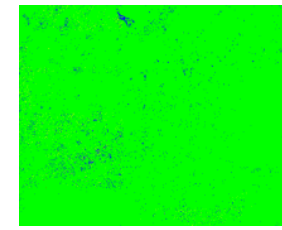

Figure (c) is PM10 map on May

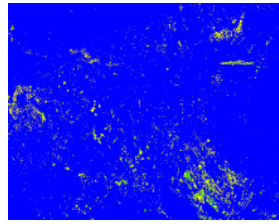

Figure (b) is vegetation map for figure (a)

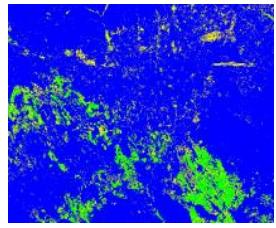

Figure (d) is vegetation map for figure (c)
Figure 4 . The relationship between vegetation and air pollution

The results of this study indicates that air pollution can be mapped using satellite information to provide a bigger area of coverage. In other words, the amount of pollution can be determined for the whole image, while if the ground stations are used, only 23 pixels of the image of the pour point is detected. Landsat- 8 image was successfully used for calculation of PM10 concentration over Tehran.

\section{REFRENCES}

Leili, M., K. Naddafi, R. Nabizadeh, M. Yunesian, and A. Mesdaghinia. "The study of TSP and PM 10 concentration and their heavy metal content in central area of Tehran, Iran." Air Quality, Atmosphere \& Health 1, no. 3 (2008): 159-166.

Beck, Hylke E., Tim R. McVicar, Albert IJM van Dijk, Jaap Schellekens, Richard AM de Jeu, and L. Adrian Bruijnzeel. "Global evaluation of four AVHRR-NDVI data sets: Intercomparison and assessment against Landsat imagery." Remote Sensing of Environment 115, no. 10 (2011): 2547-2563.

Brauer, M., Amann, M., Burnett, R.T., Cohen, A., Dentener, F., Ezzati, M., Henderson, S.B., Krzyzanowski, M., Martin, R.V., Van Dingenen, R. and Van Donkelaar, A., 2012. Exposure assessment for estimation of the global burden of disease attributable to outdoor air pollution. Environmental science \& technology, 46(2), pp.652-660.

Saleh, S.A.H. and Hasan, G., 2014. Estimation of PM10 concentration using ground measurements and Landsat 8 OLI satellite image. J Geophys Remote Sens, 3(2), pp.2169-0049.

Hadjimitsis, Diofantos G. "Aerosol optical thickness (AOT) retrieval over land using satellite image-based algorithm." Air Quality, Atmosphere \& Health 2, no. 2 (2009): 89-97.

https://www.tasnimnews.com/fa/news/1397/09/11/1889690/ 